\title{
Pulse-Spray Pharmacomechanical Thrombolysis of Native Arteriovenous Fistulas and Grafts under Ultrasound Guidance: Our Initial Experience
}

\author{
Praveen Kumar Vasanthraj ${ }^{1}$ Varun Kumar Bandi ${ }^{2}$ Venkata Sai ${ }^{1}$ Manikanthan Shekar ${ }^{2}$ \\ ${ }^{1}$ Department of Radiology, Sri Ramachandra University, Porur, \\ Chennai, Tamil Nadu, India \\ 2 Department of Nephrology, Sri Ramachandra University, Porur, \\ Chennai, Tamil Nadu, India

\begin{abstract}
Address for correspondence Praveen Kumar Vasanthraj, MD, DNB, Department of Radiology, Sri Ramachandra University, No.1 Ramachandra Nagar, Porur, Chennai-600116, Tamil Nadu, India (e-mail: doctorvpk@gmail.com).
\end{abstract}

J Clin Interv Radiol ISVIR 2017;1:20-22.

\begin{abstract}
Chronic renal diseases have been on rise with most patients requiring renal transplantation. With advances in treatment, patients undergo hemodialysis through arteriovenous (AV) fistulas and grafts. Thrombosis in these fistulas and grafts are more common and may result in failure. Interventional radiology plays an important role in managing

Keywords

- hemodialysis

- pharmacomechanical thrombolysis

- US-guided percutaneous procedure these thromboses through catheter-directed thrombolysis. With success rate of more than $80 \%$, catheter-directed thrombolysis has become the treatment of choice. However, the need of trained personnel, well-equipped catheter laboratories, and expensiveness are its disadvantages. We share our initial experience in two patients in whom we have performed ultrasound-guided percutaneous pulse-spray pharmacomechanical thrombolysis as a day-care procedure. This technique is less expensive, can have a wider outreach, and patients can be benefitted early to avoid new access.
\end{abstract}

\section{Introduction}

Catheter-guided thrombolysis have been the treatment of choice in patients with thrombolysis, However, with the advent of pharmacomechanical technique of thrombolysis using pulse-spray technique, percutaneous approach of thrombolysis have become an evolving technique under ultrasound (US) guidance, especially in hemodialysis access, and can be done on bed side or as a day-care procedure. ${ }^{1}$ Also, the need of fluoroscopy and procedure materials are reduced with this procedure, rendering it as one of the cost-effective procedures. $^{2,3}$

\section{Materials and Methods}

Patients with failed arteriovenous (AV) fistula access presented to the nephrology outpatient department. After initial evaluation, routine Doppler US scan (GE P4, Voluson, General
Electric, Boston, Massachusetts, United States) was performed to look for thrombosis. Patients with thrombosis less than 32 hours were subjected to this procedure. Preprocedure Doppler scan performed also evaluated the extent of thrombus. Patients with thrombosis extending beyond axillary vein were not included initially. After obtaining informed consent from the patient, 5,000 IU of heparin was given intravenously prior to procedure.

Using strict aseptic precautions and US guidance with linear probe, multiple 26-gauge needles were inserted in to the thrombosed segments of the veins. Needles were inserted with their tips directing toward the fistula and away from the fistula site. Once the position was confirmed within the veins, the needles were connected to three-way stop cocks with a short connector. Care was taken to avoid air bubble entering within the system.

Thrombolytic solution was prepared with dissolution of 500,000 units of Urokinase (U Frag, Bharat Serum and
DOI https://doi.org/ 10.1055/s-0036-1597840. ISSN $0000-0000$
Copyright (c) 2017 by Indian Society of Vascular and Interventional Radiology
License terms

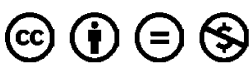


Vaccines Ltd, Ambernath, Maharashtra, India) in $20 \mathrm{~mL}$ of heparinized saline. This thrombolytic solution was taken up in 1-mL syringe and manual pulse injections were slowly given into the vein. Each injection lasts around 30 seconds to 1 minute. After that the system is flushed with heparinized saline, with care taken to not allow air bubbles within the system. Intermittent US screening was done to look for changes and progress in the thrombolysis.

The thrombolysis procedure was stopped at the end of thrombolytic solution or at demonstration of good continuous flow within the system.

Postprocedure Doppler scan was performed to assess the velocity of the flow, and the patient is followed up with another Doppler scan after 4 hours to evaluate the extent of thrombolysis. After successful thrombolysis, the patient is started on low molecular weight heparin for next 3 days to avoid any minor risk of pulmonary thromboembolism and also to maintain the patency of recanalized vessel.

Once thrombolysis has been successfully performed, it can be used for hemodialysis.

\section{Results}

\section{Patient 1}

A 58-year-old male with stage $V$ chronic kidney disease on maintenance hemodialysis through left brachiocephalic AV fistula presented with left upper limb arm swelling for 1 day with failure to access for hemodialysis. Doppler US scan showed long segment thrombus in cephalic vein (- Fig. 1) with no extension into the axillary vein and into the fistula. USguided percutaneous thrombolysis (-Fig. 2A, B) was performed as mentioned previously. Postprocedure, there was a focal thrombus in the vein with demonstrable flow in the recanalized segment. However, the focal thrombus (-Fig. 3 ) did not resolve even after 24 hours, resulting in dampened velocity necessitating the need of new fistula creation.

\section{Patient 2}

A 73-year-old patient with stage $V$ chronic kidney disease on maintenance hemodialysis through left radiocephalic fistula presented with complaints of left forearm swelling. On routine imaging, cephalic vein thrombosis was noted ( - Fig. 4A) and thrombolysis was performed ( - Fig. 4 B). Postprocedure, patient had a good flow across the segment ( $\mathbf{F i g .} \mathbf{4 C}$ ) and was subsequently successfully dialyzed.

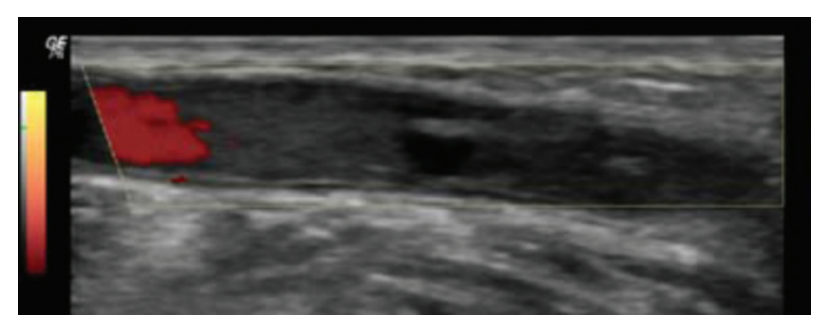

Fig. 1 A 58-year-old male with chronic kidney disease. Power Doppler ultrasound scan shows no flow in the cephalic vein.
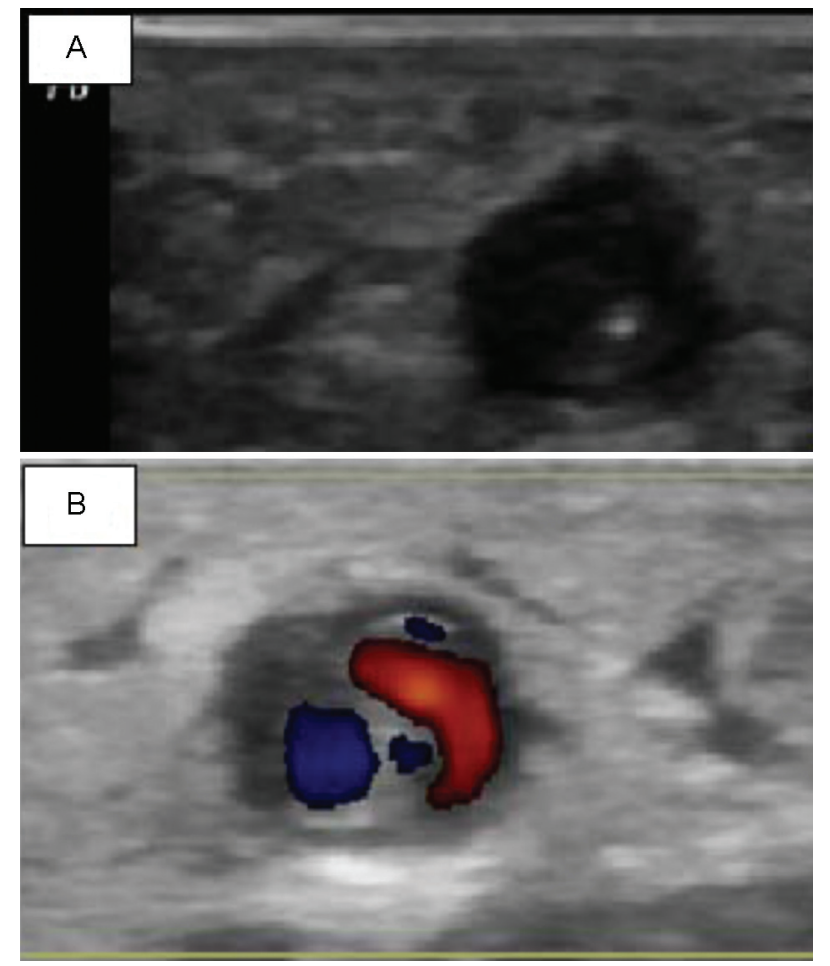

Fig. 2 (A,B) A 58-year-old man with chronic kidney disease and thrombosis of arteriovenous fistula. Ultrasound shows needle tip within cephalic vein (A) with partial recanalization during the procedure (B).

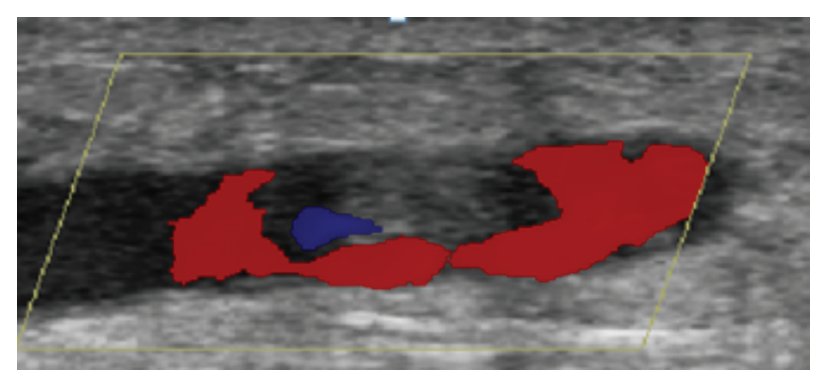

Fig. 3 A 58-year-old man with CKD and postthrombolysis. Ultrasound shows focal thrombus within the vein which did not resolve.

\section{Conclusion}

Percutaneous pharmacomechanical spray thrombolysis of thrombosed AV fistula or grafts under US guidance appears to be an evolving alternative to catheter-directed thrombolysis and can be done at a low cost and as a day-care procedure. However, more studies are needed to evaluate its safety, efficacy, and long-term results.

Conflict of Interest

None 

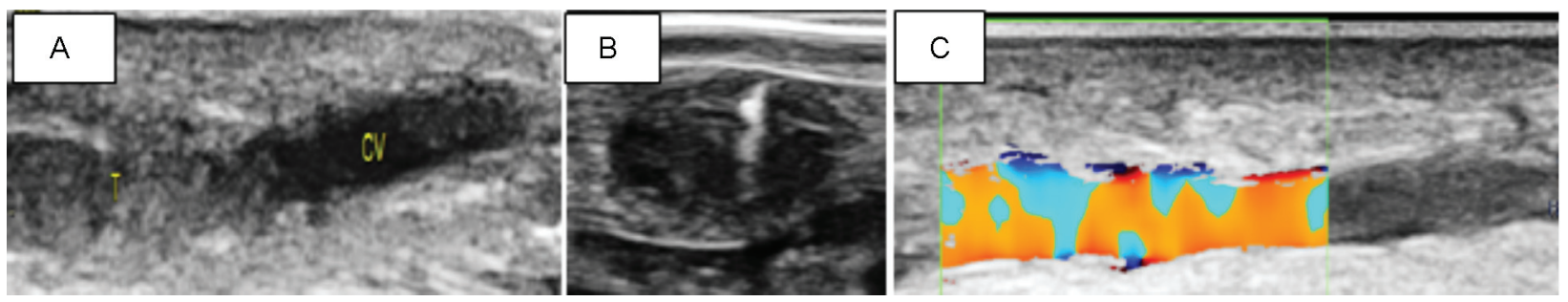

Fig. 4 (A-C) A 73-year-old patient with chronic kidney disease. (A) Ultrasound( US) shows thrombosis within the cephalic vein. (B) US-guided thrombolysis was performed with needle tip within the thrombotic vein. (C) Complete flow postthrombolysis.

\section{Disclosures}

None.

\section{Financial Support}

None.

\section{Acknowledgments}

None.

\section{References}

1 Vikrama KA, Srivalli N, Venkataramana RS. USG-guided needledirected pulse-spray pharmaco-mechanical thrombolysis of hemodialysis grafts/fistula: A novel technique. Indian J Radiol Imaging 2015;25(3):244-245

2 Cooper SG. Original report. Pulse-spray thrombolysis of thrombosed hemodialysis grafts with tissue plasminogen activator. AJR Am J Roentgenol 2003;180(4):1063-1066

3 Bookstein JJ, Fellmeth B, Roberts A, Valji K, Davis G, MachadoT. Pulsedspray pharmacomechanical thrombolysis: preliminary clinical results. AJR Am J Roentgenol 1989;152(5):1097-1100 